\title{
A Novel Method with Martingale Theory for Phase Noise Analysis in Coherent Optical Communication
}

\author{
Chengle Sui ${ }^{1}$, Qiangmin Wang ${ }^{2}$, Shilin Xiao ${ }^{1}$, Pingqing $\mathrm{Li}^{1}$ \\ ${ }^{1}$ State Key Lab of Advanced Optical Communication System and Networks, Shanghai Jiao Tong University, Shanghai, China \\ ${ }^{2}$ School of Information Security Engineering Shanghai Jiao Tong University, Shanghai, China \\ Email: sui_chengle@sjtu.edu.cn, qmwang@sjtu.edu.cn
}

Received 2013

\begin{abstract}
Phase noise has a great influence on the performance of coherent optical communication. In this paper, martingale theory is introduced to analyze the phase noise effect for the first time as far as we know. Through Fubini's Theory and martingale representation theory, we proved that $\frac{1}{T} \int_{0}^{t} \exp \left(j W_{s}\right) \cdot l(s) d s$, which denotes the phase noise effect, is a predictable martingale. Then Ito's formula for solution to stochastic differential equation is utilized for the analysis of phase noise effect. Using our method, a nonrecursive formula for the moments of phase noise is derived and signalnoise-ratio (SNR) degradation in coherent optical OFDM due to phase noise is calculated with our method.
\end{abstract}

Keywords: Martingale; Brownian Motion; Ito Itegral; Derivation of Moments; Phase Noise

\section{Introduction}

Phase noise greatly limits the performance of coherent optical communication [1], especially on the condition that recent advances in modulation formats and multisymbol detection are more and more widely introduced in optical communication system nowadays[2-3]. Therefore, the impact of phase noise has been investigated in a lot of great works. Generally, evaluating the impact of phase noises is difficult, because it's too hard to fully assess the stochastic property of laser phase noise (LPN) Wiener process and nonlinear effect induced phase noise accurately.

There are mainly two methods to evaluate phase noise effect: alternative moment method [4] and perturbation method [5]. Both of these two methods try to find a certain kind of expansion to approach the random process. Thus, the two methods will either be ineffective in some cases [6] or not accurate enough [7].

In this paper, we introduce martingale theory to analyze phase noise. Random process $\frac{1}{T} \int_{0}^{t} \exp \left(j W_{s}\right) \cdot l(s) d s$ is proved to be a predictable martingale, which is measurable with respect to proper nature filtration. Thus, the process can be substituted to Ito's formula for solution to stochastic differential equation. Then we take advantage of Ito's formula to derive a non-recursive formula for the moments of phase noise. And the SNR degradation caused by LPN is approximated through Ito isometry. These two works are examples of the application of martingale theory for the analysis of phase noise in coherent optical communication.

\section{Model and Theory}

Phase noise, frequency offset and nonlinear noise are the significant impairments of coherent optical communication. The signal before detection can be described as

$$
E_{t}=\operatorname{Re}\left\{A \cdot \exp \left[j \cdot\left(\phi_{k}+2 \pi v t+\sigma W_{t}+\phi_{N L}\right]\right\}+n_{\text {eff }}\right.
$$

where $\phi_{k}$ is the phase of modulation, $v$ represents the frequency offset and $\sigma W_{t}$ is a Wiener-Levy process which is related to LPN. The stochastic characteristics of Wiener-Levy process in a laser are known:

$$
\left\langle\sigma W_{t_{1}} \cdot \sigma W_{t_{2}}\right\rangle=\sigma^{2}\left|t_{1}-t_{2}\right|=2 \pi \beta\left|t_{1}-t_{2}\right|
$$

Here $\beta(\mathrm{Hz})$ denotes full $3 \mathrm{~dB}$ line width of laser. $\phi_{N L}$ and $n_{\text {eff }}$ corresponds to nonlinear noise and additive Gaussian noise respectively. According to [8], for the frequency offset $v \in C$, random process $2 \pi v t+\sigma W_{t}$ is a Brownian motion with drift and $\exp \left(2 \pi v t+\sigma W_{t}\right)$ denotes a geometric Brownian motion. As it is stated in [4-5], if we simply look into the impact of frequency offset and LPN, phase noise effect can be denoted as $\frac{1}{T} \int_{0}^{T} \exp \left(2 \pi v s+\sigma W_{s}\right) d s$.

Our idea starts from the equation stated in [7] 


$$
\begin{gathered}
\frac{1}{T} \int_{0}^{T} \exp \left(j W_{s}\right) \cdot l(s) d s \approx \exp \left[j \cdot \frac{1}{T} \int_{0}^{T} W_{s} \cdot l(s) d s\right] \\
{\left[\frac{1}{T} \int_{0}^{T} \exp \left(j W_{s}\right) \cdot l(s) d s\right]^{n} \approx \exp \left[j \cdot n \frac{1}{T} \int_{0}^{T} W_{s} \cdot l(s) d s\right]}
\end{gathered}
$$

In coherent optical communication systems, the symbol period is very small because of high-speed transmission. Thus, (3-4) can be quite accurate according to integral property. If the time window $l(s)$ is a rectangle over the whole symbol period, which is most frequently used in reality, we could simply to investigate the integral of a Brownian motion $Y_{t}=\frac{1}{T} \int_{0}^{t} W_{s} d s$.

$$
\text { Let } S_{t}=\exp \left[j \cdot\left(2 \pi v t+\frac{1}{T} \int_{0}^{t} \sigma W_{s} d s\right)\right] \text {. Now we will }
$$

prove that the real rand process $\frac{1}{T} \int_{0}^{t} W_{s} d s$ is a predictable martingale, and so that $S_{t}$, the geometric random process, can be adapted to Ito Formula.

Stochastic Fubini's Theorem: The stochastic process $W_{s}$ is a martingale with respect to a filtration $\left\{\Gamma_{t}\right\}_{t \geq 0^{-}}$ and probability measure $\mathrm{P}$. Then the underlying filtration probability space can be represented as $\left(\Omega, \Gamma,\left\{\Gamma_{t}\right\}_{t \geq 0^{-}}, \mathrm{P}\right)$. If $\Phi(t, r, w): R_{+} \times R_{+} \times \Omega \rightarrow R_{+}$is a bounded random variable, and is predictable for filtration $\left\{\Gamma_{t}\right\}_{t \geq 0^{-}}$, then for $\forall T>0$

$$
\int_{0}^{t} \int_{R} \Phi(s, r, w) l_{[0, T]}(r) d r d W=\int_{R} \int_{0}^{t} \Phi(s, r, w) l_{[0, T]}(r) d W_{s} d r
$$

Utilizing (10), yielding

$$
\begin{aligned}
Y_{t} & =\frac{1}{T} \int_{0}^{t} W_{s} d s=\frac{1}{T} \int_{0}^{t} \int_{0}^{s} 1 d W_{s} d s \\
& =\frac{1}{T} \int_{0}^{t} \int_{s}^{t} 1 d s d W_{s}=\frac{1}{T} \int_{0}^{t}(t-s) d W_{s}
\end{aligned}
$$

Now the equation above can be utilized to prove $\frac{1}{T} \int_{0}^{t} W_{s} d s$ is a predictable martingale.

Martingale Representation Theorem: Let $W_{s}$ be a Brownian motion on a standard filtered probability space $\left(\Omega, \Gamma,\left\{\Gamma_{t}\right\}_{t \geq 0^{-}}, P\right)$ if $\mathrm{M}_{\mathrm{t}}$ is a square integrable random variable measurable with respect to $\left\{\Gamma_{t}\right\}_{t \geq 0^{-}}$, then there exists a predictable process $\vartheta_{\mathrm{t}}$ which is adapted to $\left\{\Gamma_{t}\right\}_{t \geq 0^{-}}$such that

$$
M_{t}=M_{0}+\int_{0}^{t} \vartheta_{s} d s
$$

Therefore, the stochastic process $\frac{1}{T} \int_{0}^{t} W_{s} d s$ (i.e. $\frac{1}{T} \int_{0}^{t} W_{s} d s$ according to (16)) can be represented by Ito integral, which is called as predictable representation property[13]. It means the geometric random process $S_{t}$ can also be a solution of Ito's formula.

Ito's Formula: let partial derivative of function exits and is continuous, then

$$
\begin{aligned}
d f\left(t, W_{t}\right)= & f^{\prime}\left(t, W_{t}\right) d W_{t}+\dot{f}\left(t, W_{t}\right) d t \\
& +\frac{1}{2} f^{\prime \prime}\left(t, W_{t}\right) d t
\end{aligned}
$$

By substituting $f$ with $S_{t}$, yielding

$$
d S_{t}=j \frac{t}{T} \sigma S_{t} d W_{t}+\left(j \cdot 2 \pi v-\frac{t^{2}}{2 T^{2}}\right) \cdot S_{t} d t
$$

\section{Applications}

In the section, the analysis above will be used to introduce some meaningful utilization in optical coherent communication, including moments of phase noise, SNR degrade due to LPN. These discussions here will reveal the way to apply martingale theory for analyzing phase noise effect.

\subsection{Non-recursive Formula for the Moments of Phase Noise}

Reference [9] proved a recursive formula for the moments of phase noise. Method of moments is very important in performance evaluation of digital communication systems [6]. Here a different approach is revealed to get a non-recursive formula for the moments of phase noise. By substituting (4) into (6), a new stochastic differential equation can be denoted as

$$
d S_{t}^{n}=j \cdot \frac{n \sigma t}{T} S_{t}^{n} d W_{t}+\left(j \cdot 2 \pi n v-\frac{t^{2}}{2 T^{2}} n(n-1) \sigma^{2}\right) \cdot S_{t}^{n} d t
$$

Integrating and then taking expectation on both side of the equation, yielding

$$
E\left[S_{t}^{n}\right]-E\left[S_{0}^{n}\right]=\int_{0}^{t}\left(j \cdot 2 \pi n v-\frac{1}{2 T^{2}} n(n-1) \sigma^{2} s^{2}\right) E\left[S_{s}^{n}\right] d s
$$

Since the expectation of Wiener process is zero, by solving the integral equation (11), we can get

$$
\begin{gathered}
\begin{aligned}
E\left[S_{t}^{n}\right] & \approx S_{0}^{n} \exp \left[\left(j \cdot 2 \pi n v-\frac{1}{2 T^{2}} n(n-1) \sigma^{2} t^{2}\right) t\right] \\
& =\exp \left[\left(j \cdot 2 \pi n v-\frac{\pi n(n-1) \beta t^{2}}{T^{2}}\right) t\right]
\end{aligned} \\
\text { (Note: } \left.S_{0}=\exp \left[j \cdot\left(2 \pi v t-\frac{1}{T} \int_{0}^{t} \sigma W_{s} d s\right)\right]_{t=0}=1\right)
\end{gathered}
$$

The result is much simpler than that of [9], and it's easy for calculation. Figure 1 shows the first three mo 


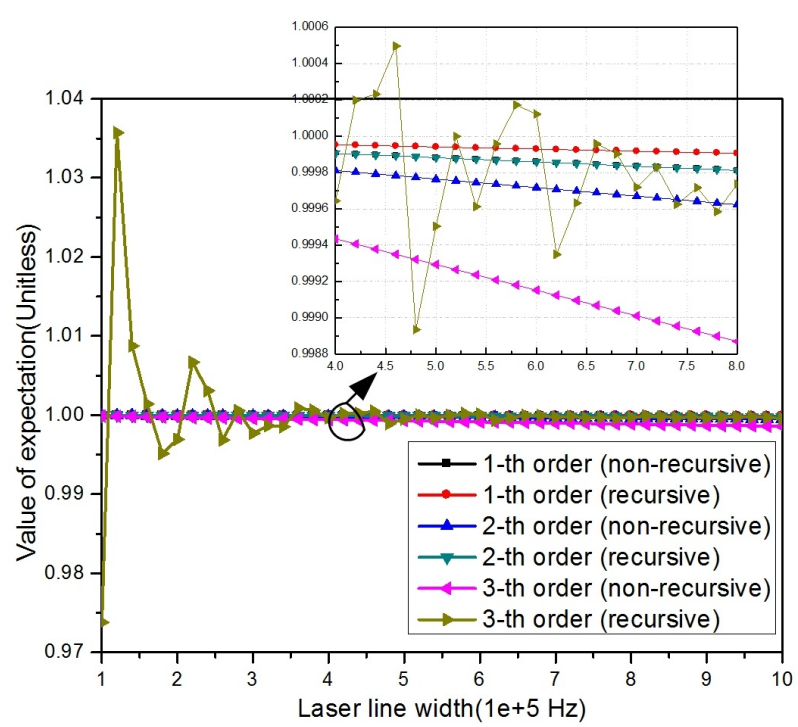

Figure 1. The first three moments as function of laser line width.

ments as function of laser line width. We can observe that the expectation value of both recursive and non-recursive formula is almost the same except that the third moments of recursive solution suffers fierce shock when laser line width is below $400 \mathrm{kHz}$. It's quite unreasonable because phase noise effect should be smaller if laser line width degrades. Therefore, the non-recursive formula is more effective than the recursive formula given by [9].

\subsection{SNR Degradation Due to Phase Noise in Optical OFDM}

Coherent optical OFDM systems are very sensitive to the phase noise of laser. The impact of phase noise in wireless OFDM has been widely evaluated, including [10-11]. Here, we show a totally different method to give an evaluation on phase noise effect in optical OFDM system. As it's widely known, if we simply investigate the effect of phase noise, the signal before deciding could be written as

$$
x_{k}=a_{k} I_{0}+n_{k, t}
$$

where $I_{0}=\frac{1}{T} \int_{0}^{T} \exp \left(j W_{s}\right) d s$, and the SNR degradation duo to phase noise can be denoted by [10]

$$
D \approx \frac{10}{\ln 10}\left(1-E_{0}^{2}\right)(1+S N R)
$$

Thus, the key point is to calculate $E_{0}=E^{2}\left(\left|I_{0}\right|\right)$. Expand (3) by Taylor series, yielding

$$
\frac{1}{T} \int_{0}^{T} \exp \left(j W_{s}\right) d s \approx 1+\frac{j}{T} \int_{0}^{T} W_{s} d s-\frac{1}{2 T} \int_{0}^{T} W_{s}^{2} d s
$$

Therefore, we can firstly get

$$
\begin{aligned}
E\left(\left|I_{0}\right|^{2}\right) & =E\left[\left(1-\frac{1}{2 T} \int_{0}^{T} W_{s}^{2} d s\right)^{2}+\left(\frac{1}{T} \int_{0}^{T} W_{s} d s\right)^{2}\right] \\
& \approx 1-E\left(\frac{1}{T} \int_{0}^{T} W_{s}^{2} d s\right)+E\left[\left(\frac{1}{T} \int_{0}^{T} W_{s} d s\right)^{2}\right]
\end{aligned}
$$

Now Ito isometry can be applied to calculate (15). Ito isometry: if $\mathrm{f}$ is an elementary function, then

$$
E\left[\left(\int_{0}^{t} f(s, w) d W_{s}\right)^{2}\right]=\int_{0}^{t} E\left[f(s, w)^{2}\right] d s
$$

Therefore, the derivation for the mean variance of is

$$
\begin{aligned}
E\left[Y_{t}^{2}\right] & =E\left[\left(\frac{1}{T} \int_{0}^{T}(t-s)\right) d W_{s}\right] \\
& =\frac{1}{T^{2}} \int_{0}^{t}(t-s)^{2} d s=\frac{1}{3 T^{2}} t^{3}
\end{aligned}
$$

So (16) can be simplified as $E\left(\left|I_{0}\right|^{2}\right)=1-\frac{1}{6} \sigma^{2} T$. Similarly, we can get $\sigma^{2}\left(\left|I_{0}\right|\right)=\frac{1}{60} \sigma^{2} T$. And from Ito's Formula, it's easy to get that $E W_{s}^{2}=\sigma^{2} s$. So the parameter will be $E_{0}^{2}=E\left(\left|I_{0}\right|^{2}\right)-\sigma^{2}\left(\left|I_{0}\right|\right)$.

Substituting $E_{0}^{2}$ into (14), the SNR degradation due to phase noise in coherent optical OFDM can be expressed by

$$
D \approx \frac{11}{6 \ln 10}(2 \pi N \beta T) \cdot S N R
$$

The result fits well with [10], yet the method is much simpler. Figure 2 shows the relationship between laser width and SNR degradation when the transmission rate is set at $40 \mathrm{Gbps}$ with 64 carrier frequencies. If BER is set to be $10^{-7}$, the value of SNR for M-QAM and M-PSK are approximated by $10(M-1)$ and $15 / \sin ^{2}(\pi / M)$ [10].

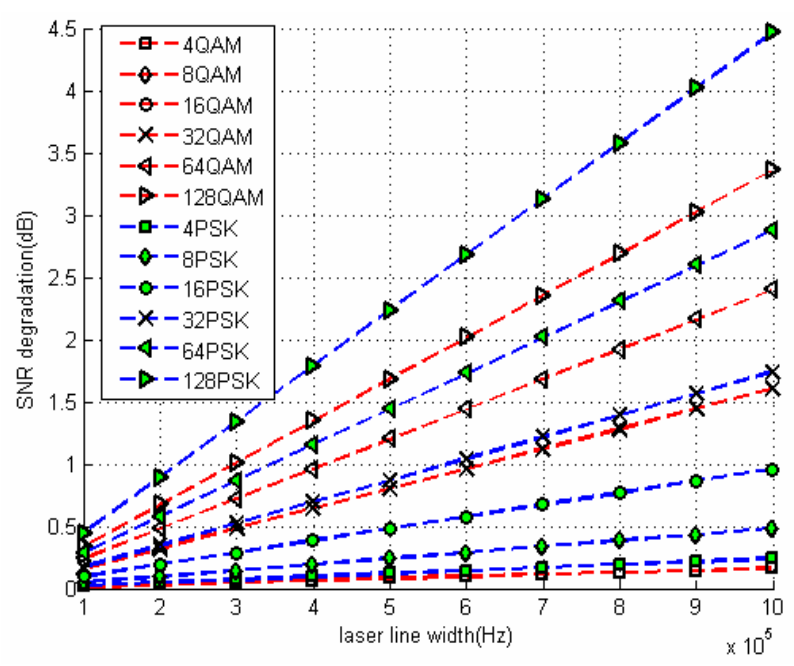

Figure 2. SNR degradation as function of laser line width. 
It's easy to observe that MPSK modulated signal suffers more SNR degradation due to phase noise. And with the increasing of signal constellation's size, the signal becomes more and more sensitive to the phase noise. If the laser line width is $1 \mathrm{MHz}$ and the modulation format is 64QAM, extra 2.5dB SNR needs to be compensated for the phase noise effect.

\section{Conclusions}

In the paper, Martingale theory was introduced to analyze the effect of phase noise for the first time. Through stochastic Fubini's theorem and martingale representation theorem, we proved the process $\frac{1}{T} \int_{0}^{t} W_{s} d s$ is a predictable martingale which can be applied to Ito Formula. Then we applied our theory to the derivation of a nonrecursive formula for the moments of phase noise, which is accurate, yet much simpler than its recursive style given by [9]. At last, we evaluated the performance of coherent optical communication as a function of laser line width by Ito isometry. The two examples reveal the way to apply martingale theory for the analysis of phase noise.

\section{Acknowledgements}

The work was jointly supported by the National 973 Project of China (No. 2012CB315602), the National Nature Science Fund of China (No.61271216 and No. 60972032), and the National 863 Hi-tech Project of China (No. 2011AA01A106).

\section{REFERENCES}

[1] K. Kikuchi, "Characterization of Semiconductor-Laser Phase Noise and Estimation of Bit-Error Rate Performance with Low-Speed Offline Digital Coherent Receivers," Optics Express, Vol.20, 2012, pp. 5291-5302. doi:10.1364/OE.20.005291

[2] S. Jansen, D. van den Borne and M. Kuschnerov, "Advances in Modulation Formats for Fiber-Optic Transmission Systems," in CLEO:2011 Laser Applications to
Photonic Applications, OSA Technical Digest (CD) (Optical Society of America, 2011), paper CWJ1.

[3] M. Nazarathy, X. Liu, L. Christen, Y. Lize and A. Willner, "Self-Coherent Multisymbol Detection of Optical Differential Phase-Shift Keying," Journal of Lightwave Technology, Vol. 26, 2008, pp. 1921-1934. doi:10.1109/JLT.2007.912055

[4] G. L. Pierobon and L. Tomba, "Moment Characterization of Phase Noise in Coherent Optical Systems," Journal of Lightwave Technology, Vol. 9, No. 8, 1991, pp. 996-1005. doi:10.1109/50.84166

[5] G. J. Foschini and G. Vannucci, "Characterizing Filtered Light Waves Corrupted by Phase Noise,” IEEE Transactions on Information Theory, Vol. 34, No. 6, 1988, pp. 1437-1448. doi:10.1109/18.21283

[6] C. P. Kaiser, M. Shafi and P. J. Smith, “Analysis Methods for Optical Heterodyne DPSK Receivers Corrupted by Laser Phase Noise,” Journal of Lightwave Technology, Vol. 11, 1993, pp. 1820-1830. doi:10.1109/50.251180

[7] Y. Atzmon and M. Nazarathy, "Laser Phase Noise in Coherent and Differential Optical Transmission Revisited in the Polar Domain,” Journal of Lightwave Technology, Vol. 27, 2009, pp. 19-29. doi:10.1109/JLT.2008.925036

[8] A. Etheridge, “A Course in Financial Calculus,” Cambirdge University Press, Cambridge, 2002. doi:10.1017/CBO9780511810107

[9] I. T. Monroy and G. Hooghiemstra, "On A Recursive Formula for the Moments of Phase Noise," IEEE Transactions on Communications, Vol. 48, No. 6, 2000, pp.917-920. doi:10.1109/26.848548

[10] T. Pollet, M. V. Blade and M. Moeneclaey, "BER Sensitivity of OFDM Systems to Carrier Frequency Offset and Wiener Phase Noise" IEEE Transactions on Communications, Vol. 43, No.2, pp. 191-193,

[11] L. Tomba, "On the Effect of Wiener Phase Noise in OFDM Systems," IEEE Transactions on Communications, Vol. 46, No. 5, 1998, pp. 580-583. doi:10.1109/26.668721

[12] K. L. Chung and R. Williams, "Introduction to Stochastic Integration,” 2nd Edition, Birkhauser Press, Berlin, 1990. doi:10.1007/978-1-4612-4480-6

[13] D. Revuz and M. Yor, "Continuous Martingales and Brownian Motion,” Springer Press, Berlin, 2004. 